ISSN electrónico: 2445-1355

DOI: https://dx.doi.org/10.14201/fj2020521526

\title{
FARMACOCINÉTICA POBLACIONAL DE FENITOÍNA EN PACIENTES ADULTOS
}

\section{Population Pharmacokinetics of Phenytoin in Adult Patients}

Carlos TAPIA ARTILES; J Samuel PÉREZ-BLANCO; Dolores SANTOS BUELGA; María José GARCÍA SÁNCHEZ*

Departamento de Ciencias Farmacéuticas. Facultad de Farmacia. Licenciado Méndez Nieto, sn. 37003 Salamanca, España

*Autor correspondiente: MJ García Sánchez, Departamento de Ciencias Farmacéuticas. Facultad de Farmacia. Licenciado Méndez Nieto, sn. 37003

Salamanca, España

Correo-e: mjgarcia@usal.es

RESUMEN: La fenitoína (DPH) es un antiepiléptico considerado de primera elección en el tratamiento de las crisis focales y convulsiones tónico-clónicas. DPH presenta elevada unión a proteínas plasmáticas, un comportamiento cinético no lineal dependiente de la dosis, elevada variabilidad cinética interindividual y un estrecho margen terapéutico; estas características aconsejan su monitorización para optimizar su balance eficacia/toxicidad. El objetivo de este trabajo fue la caracterización de la farmacocinética de DPH en población adulta.

Concentraciones en estado de equilibrio de DPH procedentes de 215 pacientes adultos fueron utilizadas para desarrollar un modelo farmacocinético poblacional (PopPK) aplicando una metodología de efectos mixtos no lineales con el programa NONMEM v.7.3.

La cinética de DPH fue descrita adecuadamente mediante un modelo monocompartimental con absorción de orden cero y cinética de eliminación no lineal ajustada a la ecuación de Michaelis-Menten. Edad, peso, magnitud de dosis y comedicación con ácido valproico han mostrado influencia sobre la velocidad de eliminación de DPH, reduciendo su variabilidad de forma considerable.

Ediciones Universidad de Salamanca / @@ఠ $\quad$ FarmaJournal, vol. 5, núm. 2 (2020), pp. 15-26 
Se ha obtenido un modelo PopPK preliminar que ha mostrado una adecuada capacidad descriptiva y predictiva en población adulta en un amplio rango de dosis (50-800 mg/día). No obstante, se precisa su aplicación en el contexto clínico para confirmar su validez.

Palabras clave: fenitoína; farmacocinética poblacional; monitorización de fármacos.

ABSTRACT: Phenytoin (DPH) is an antiepileptic drug used in the first line of focal seizures and tonic-clonic seizures treatments. Phenytoin shows a highly protein binding, non-lineal dose-dependent kinetics, large pharmacokinetic variability and narrow therapeutic index; reasons why this antiepileptic is commonly monitored to optimize the efficacy/ toxicity balance. The aim of the present work was to characterize the DPH pharmacokinetics in adult population.

Drug plasma levels at steady-state from 215 adult patients were used to develop a population pharmacokinetic model (PopPK) using a non-linear mixed effect modelling approach with NONMEM v.7.3.

Pharmacokinetics of DPH were described following one-compartment distribution model with zero-order absorption and Michaelis-Menten non-lineal saturable elimination. Age, weight, magnitude of DPH dose and co-medication with valproic acid have been shown influence on maximum velocity of PHT elimination, significantly decreasing the variability of this pharmacokinetic parameter.

This preliminary PopPK developed has shown an adequate evaluation of the descriptive and predictive performance in adult population within a large dose range $(50-800 \mathrm{mg} /$ day). However, its implementation in the clinical setting is required to confirm its suitability.

Keywords: phenytoin; population pharmacokinetics; therapeutic drug monitoring.

\section{INTRODUCCIÓN}

La fenitoína (DPH) es un fármaco antiepiléptico clásico, considerado de primera elección en el tratamiento de las crisis focales y convulsiones tónicoclónicas, que se administra por vía oral e intravenosa, principalmente (Vajda, Eadie, 2014). 
Administrado por vía oral presenta un proceso de absorción de orden cero con una velocidad media en pacientes adultos de $50 \mathrm{mg} / \mathrm{hora}$, por lo que el tiempo necesario para alcanzar las concentraciones máximas $\left(\mathrm{t}_{\max }\right)$ depende de la dosis administrada. Su absorción, además, se ve afectada por trastornos gastrointestinales y por el uso de antiácidos (Vajda, Eadie, 2014; Winter, 1994).

Este antiepiléptico se distribuye ampliamente en el organismo (volumen aparente de distribución, $\mathrm{Vd}, 0,6-1 \mathrm{~L} / \mathrm{kg}$ ), accediendo al sistema nervioso central gracias a su elevada liposolubilidad. La DPH se une a la albúmina aproximadamente un $90 \%$, por lo que pequeñas variaciones en la concentración de esta proteína pueden originar grandes cambios en la fracción libre de fármaco en plasma (Vajda, Eadie, 2014; Winter, 1994).

La DPH se elimina mayoritariamente por metabolismo hepático oxidativo, principalmente mediado por las isoenzimas CYP2C9 y CYP2C19, con capacidad de saturación a los niveles que alcanza con las dosis habitualmente administradas, presentando un comportamiento cinético no lineal dependiente de la dosis, que se ajusta adecuadamente a la ecuación de Michaelis-Menten (Eadi, 2002; Vajda, Eadie, 2014; Winter, 1994).

El estrecho margen terapéutico que presenta la DPH (10-20 mg/L) junto con la amplia variabilidad farmacocinética inter- e intraindividual justifican la monitorización de sus niveles plasmáticos con el fin de optimizar su posología. Para ello se extrae al paciente una muestra de sangre una vez se haya alcanzado el estado de equilibrio y habitualmente al final del intervalo de dosificación, obteniendo así una concentración mínima $\left(\mathrm{C}_{\min }{ }^{\text {ss }}\right)$ de gran utilidad para el ajuste de las dosis (Gugler et al., 1976).

El objetivo de este trabajo es desarrollar un modelo farmacocinético poblacional (PopPK, Population Pharmacokinetics) preliminar de DPH administrada por vía oral en pacientes adultos evaluando la posible influencia en la cinética de factores demográficos, de diagnóstico y medicación antiepiléptica concomitante con carbamazepina (CBZ), ácido valproico (VLP) y fenobarbital (FB).

\section{MATERIALES y MÉTODOS}

\subsection{Pacientes y recogida de datos}

Estudio retrospectivo que utiliza datos clínicos recogidos de la monitorización de niveles plasmáticos (TDM, Therapentic Drug Monitoring) de DPH en la unidad de Farmacocinética Clínica del Servicio de Farmacia del Hospital Universitario de Salamanca durante el periodo comprendido entre enero de 1992 y marzo de 2013.

Para la selección de pacientes se han utilizado como criterios de inclusión: información completa de sus características demográficas, pautas de dosificación 
de DPH, tiempos de muestreo, comedicación y diagnóstico, excluyéndose aquellos pacientes en los que existen sospechas de falta de adherencia al tratamiento -aplicación del criterio del coeficiente de variación (CV, \%) del índice nivel/dosis-, y cuando la administración de DPH fue por vía intravenosa. Tampoco se han incluido pacientes que recibían concomitantemente rifampicina, lamotrigina o levetiracetam, o presentaban alteraciones en la albumina ya que su baja incidencia en la muestra inicialmente revisada impedía evaluar adecuadamente su influencia en la variabilidad farmacocinética de DPH. Las características demográficas y clínicas de los 215 pacientes incluidos en el estudio se presentan en la Tabla 1.

Las muestras de plasma se obtuvieron habitualmente al final del intervalo de dosificación y una vez alcanzada la situación de equilibrio $\left(\mathrm{C}_{\min }\right.$ ss $)$ y fueron analizadas por una técnica de inmunoensayo quimioluminiscente de micropartículas (CMIA) (Abbott®).

\subsection{Análisis farmacocinético}

El análisis PopPK se realizó aplicando una metodología fármaco-estadística basada en modelos de efectos mixtos no lineales (NLME, nonlinear mixed-effects modelling). Esta aproximación matemática considera parámetros farmacocinéticos de efectos fijos, así como de efectos aleatorios, que cuantifican la magnitud y distribución de la variabilidad interindividual de los parámetros farmacocinéticos y variabilidad residual. El desarrollo del modelo se llevó a cabo con el programa comercial NONMEM v 7.3. Para el procesado de los resultados incluyendo test estadísticos adicionales y representación gráfica de los resultados se utilizó el software libre R versión 3.5.1.

Los modelos estructurales previamente descritos en la literatura y evaluados en el presente trabajo fueron:

$$
\begin{aligned}
& v_{0}=\frac{v_{\max } \cdot C}{k_{m}+C} \\
& v_{0}=\frac{v_{\max } \cdot C}{k_{m}+C}+C l \\
& v_{0}=\frac{v_{\max \cdot[D / V d]}}{k_{m}+[D / V d]} \\
& v_{0}=\frac{v_{\max } \cdot[D / V d]}{k_{m}+[D / V d]}+C l
\end{aligned}
$$

Ecuación 1

Ecuación 2

Ecuación 3

Ecuación 4

Donde $\mathrm{V}_{0}$, velocidad de eliminación; $\mathrm{V}_{\max }$, velocidad máxima de eliminación; $\mathrm{K}_{\mathrm{m}}$, concentración con la cual la velocidad de eliminación alcanza un valor igual 
Tabla 1. Características de los pacientes incluidos en el estudio

\begin{tabular}{|c|c|c|c|c|c|}
\hline Variable & MT, media [rango] & \begin{tabular}{|c|} 
CD, media \\
{$[$ rango $]$}
\end{tabular} & $\begin{array}{l}\text { DV, media } \\
\text { [rango }]\end{array}$ & \multicolumn{2}{|c|}{$\begin{array}{l}\text { DF, media } \\
\text { [rango] }\end{array}$} \\
\hline Edad (años) & $\begin{array}{c}58 \\
{[19-87]}\end{array}$ & $\begin{array}{c}48 \\
{[18-82]}\end{array}$ & $\begin{array}{c}50 \\
{[19-84]}\end{array}$ & \multicolumn{2}{|c|}{$\begin{array}{c}51 \\
{[23-91]}\end{array}$} \\
\hline Altura $(\mathrm{cm})$ & $\begin{array}{c}162 \\
{[140-180]}\end{array}$ & $\begin{array}{c}163 \\
{[145-180]}\end{array}$ & $\begin{array}{c}163 \\
{[145-177]}\end{array}$ & \multicolumn{2}{|c|}{$\begin{array}{c}165 \\
{[140-182]}\end{array}$} \\
\hline Peso (kg) & $\begin{array}{c}67 \\
{[45-97]}\end{array}$ & $\begin{array}{c}70 \\
{[40-99]}\end{array}$ & $\begin{array}{c}69 \\
{[50-99]}\end{array}$ & \multicolumn{2}{|c|}{$\begin{array}{c}69 \\
{[48-93]}\end{array}$} \\
\hline $\begin{array}{c}\text { Dosis diaria dph } \\
(\mathrm{mg})\end{array}$ & $\begin{array}{c}284 \\
{[140-400]}\end{array}$ & $\begin{array}{c}279 \\
{[50-550]}\end{array}$ & $\begin{array}{c}325 \\
{[50-800]}\end{array}$ & \multicolumn{2}{|c|}{$\begin{array}{c}314 \\
{[100-600]}\end{array}$} \\
\hline$[\mathrm{Dph}](\mu \mathrm{g} / \mathrm{ml})$ & $\begin{array}{c}12,05 \\
{[1,94-44,00]}\end{array}$ & $\begin{array}{c}12,76 \\
{[0,68-45,46]}\end{array}$ & $\begin{array}{c}10,2 \\
{[1,28-30,36]}\end{array}$ & \multicolumn{2}{|c|}{$\begin{array}{c}13,47 \\
{[2,37-37,08]}\end{array}$} \\
\hline N. ${ }^{\circ}$ pacientes & 76 & 48 & 44 & \multicolumn{2}{|c|}{48} \\
\hline $\begin{array}{c}\text { SEXO } \\
{[\text { hombres (\%)] }}\end{array}$ & 53 & 49 & 64 & \multicolumn{2}{|c|}{85} \\
\hline \multicolumn{6}{|l|}{ Diagnósticos ( \%) } \\
\hline \multirow{4}{*}{$\begin{array}{c}\text { CRISIS } \\
\text { EPILEPTICAS }\end{array}$} & $\begin{array}{c}\text { FOCAL INICIO } \\
\text { MOTOR }\end{array}$ & 17,8 & 17,0 & 23,0 & 17,8 \\
\hline & $\begin{array}{l}\text { GENERALIZADA } \\
\text { INICIO MOTOR }\end{array}$ & 0 & 4,3 & 2,6 & 6,7 \\
\hline & $\begin{array}{l}\text { GENERALIZADA } \\
\text { INICIO } \\
\text { DESCONOCIDO }\end{array}$ & 1,8 & 0 & 0 & 4,4 \\
\hline & $\begin{array}{l}\text { EPILEPSIA SIN } \\
\text { CLASIFICAR }\end{array}$ & 46,4 & 63,8 & 56,4 & 53,3 \\
\hline TUMORES* & 1,8 & 0 & 2,6 & \multicolumn{2}{|c|}{4,4} \\
\hline OTROS* $*$ & 32,1 & 14,9 & 15,4 & \multicolumn{2}{|c|}{13,3} \\
\hline \multicolumn{6}{|c|}{$\begin{array}{l}\text { *Tumores (se incluye cualquier tipo de neoplasia/tumor cerebral). } \\
\text { *Otros (incluye neurocirugía, absceso cerebral, accidente cerebrovascular, traumatismo cerebral, neu- } \\
\text { ropatía, glioblastoma, hemorragia cerebral...). } \\
\text { MT, monoterapia de fenitoína (DPH); DC, biterapia con carbamazepina; DV, biterapia con ácido val- } \\
\text { proico; DF, biterapia con fenobarbital. }\end{array}$} \\
\hline
\end{tabular}

a la mitad de la velocidad máxima; C, concentración plasmática; D, dosis; Vd, volumen de distribución; CL, aclaramiento plasmático.

Los criterios de selección de modelos se basaron en la aplicación del test de verosimilitud (log-likelihood ratio test), asumiendo una distribución $\chi^{2}$ con una significación estadística a $=0,05$ (excepto en la selección de factores pronóstico que fue más restrictiva, $a=0,01$ ) y la bondad de ajuste de los datos observados frente a las predicciones poblacionales e individuales. 
Adicionalmente, para evaluar la capacidad predictiva y aplicabilidad del modelo PopPK desarrollado, se compararon las predicciones individuales frente a las observaciones de las concentraciones plasmáticas de DPH estratificadas por cuartiles para cada una de las variables incluidas en el modelo. Finalmente, se desarrollaron simulaciones determinísticas de manera univariante con el fin de evaluar el impacto y la relevancia clínica de los factores pronóstico seleccionados e incluidos en el modelo final.

\section{Resultados}

Los datos de concentración plasmática de DPH analizados se ajustan adecuadamente a un modelo farmacocinético monocompartimental con absorción de orden cero y eliminación de tipo no lineal ajustada a una cinética de Michaleis-Menten (ecuación 1) caracterizada por los parámetros $\mathrm{V}_{\max } \mathrm{y}_{\mathrm{m}}$. No obstante, el tipo de datos obtenidos con un único punto por régimen de dosificación hizo necesaria la fijación del volumen aparente de distribución $(\mathrm{Vd})$ y la $\mathrm{K}_{\mathrm{m}}$ a valores de $0,61 \mathrm{~L} / \mathrm{Kg}$ y $5,33 \mathrm{mg} / \mathrm{L}$ respectivamente, obtenidos de la literatura (Alqahtani et al., 2019).

La Figura 1 muestra las $\mathrm{C}_{\min }$ ss $\mathrm{DPH}$ observadas para 4 rangos de dosis diaria: 50-200; 200-300; 300-400, y > 400 mg en 4 situaciones distintas: monoterapia (MT), biterapia con CBZ (CD), con VLP (DV) y con FB (DF). Se observa que las concentraciones de DPH en equilibrio son similares y están dentro o próximas al margen terapéutico aceptado para este fármaco $(10-20 \mathrm{mg} / \mathrm{L})$ para los 4 rangos de dosis considerados. Ello pone de manifiesto la elevada variabilidad farmacocinética de DPH y la utilidad de la TDM para este fármaco, que para conseguir niveles terapéuticos puede requerir la administración de dosis muy diferentes.

FIgURA 1: Concentraciones mínimas de DPH en estado de equilibrio $\left(\mathrm{C}_{\min }\right.$ ss $)$ estratificadas en 4 rangos de dosis/día y en función del tipo de tratamiento recibido

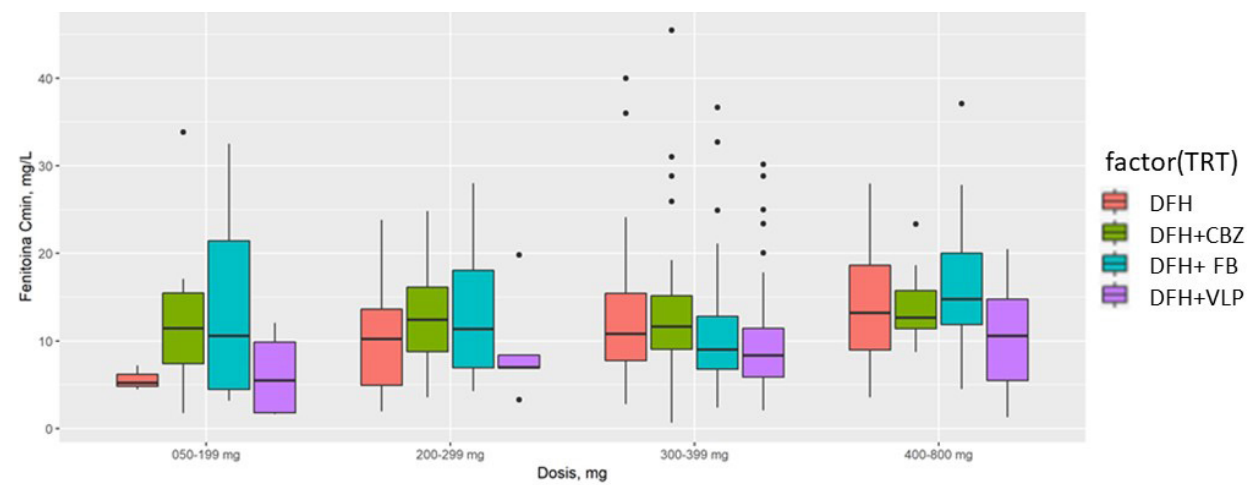


Una vez ajustados los datos al modelo estructural de Michaelis-Menten, se procedió a analizar la influencia de las covariables: edad, peso, sexo y comedicación con CBZ, VLP y FB, aplicando para ello los criterios estadísticos previamente descritos. Las covariables que mostraron influencia significativa sobre $\mathrm{V}_{\max }$ fueron la edad, el peso y la comedicación con VLP. La inclusión de estas covariables redujo la función mínima objetivo en 230 unidades y la variabilidad interindividual de este parámetro en aproximadamente un $55 \%$. El modelo final para $\mathrm{V}_{\max }$ presentó la siguiente estructura:

$$
\mathrm{V}_{\max }(\mathrm{mg} / \mathrm{h})=\Theta_{1-4} \times\left[1+\Theta_{5} \times(\text { Peso-59.5) }] \times\left[1-\Theta_{6} \times(\text { Edad-55.5) }] \times\left(1+\Theta_{7}\right)^{\mathrm{VLP}}\right.\right.
$$

Los valores de los parámetros de efectos fijos $\left(\Theta_{\mathrm{n}}\right)$ y aleatorios estimados para el modelo final se muestran en la Tabla 2.

\section{Tabla 2. Parámetros estimados en el Modelo PopPK final para DPH administrada vía oral}

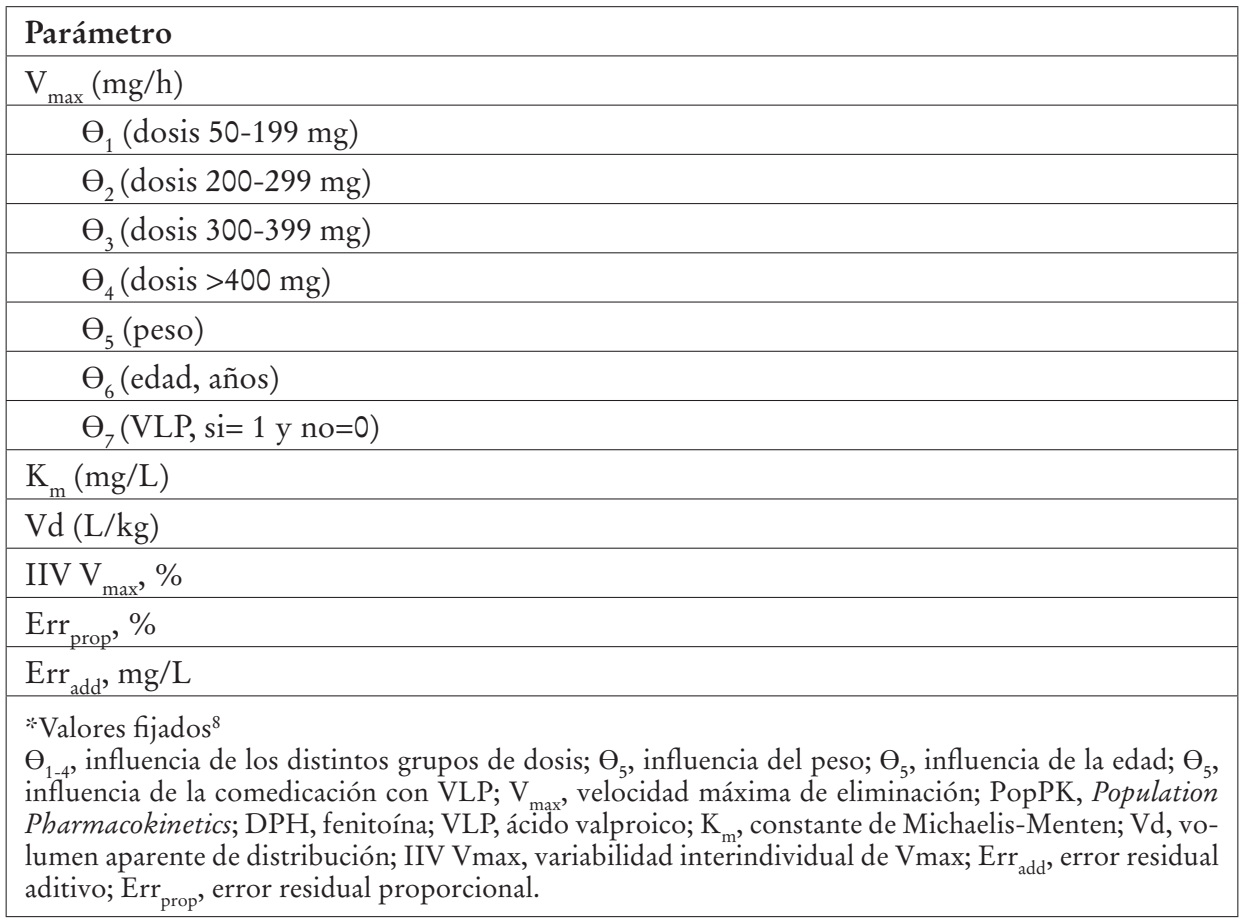


La bondad del ajuste del modelo PopPK final se muestra en la Figura 2, que recoge la correlación entre las concentraciones de DHF poblacionales predichas (PRED) y las individuales (IPRED) con las observadas y los residuales ponderados frente al tiempo observándose la ausencia de un sesgo significativo.

La adecuada capacidad predictiva del modelo se pone de manifiesto comparando las concentraciones plasmáticas de DPH observadas con las predichas por el modelo PopPK desarrollado considerando cada una de la covariables incluidas: dosis, peso, edad y comedicación con VLP (Figura 3).

La Figura 4 muestra las simulaciones determinísticas de la evolución de las concentraciones de DPH teniendo en cuenta diferentes escenarios de dosis, edad, peso y comedicación con VLP.

Figura 2. Gráficos de bondad de ajuste del modelo PopPK: [DPH]:

Regresión local
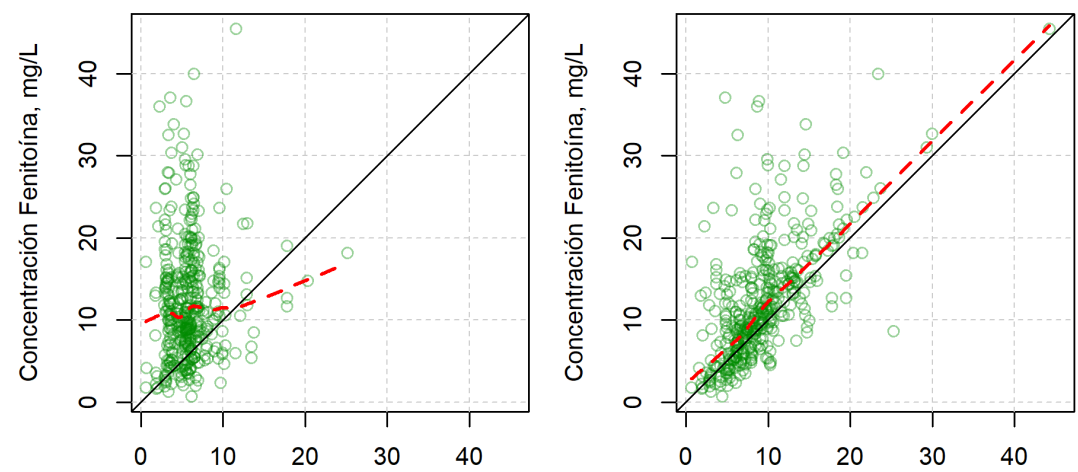

Predicción poblacionale, $\mathrm{mg} / \mathrm{L}$

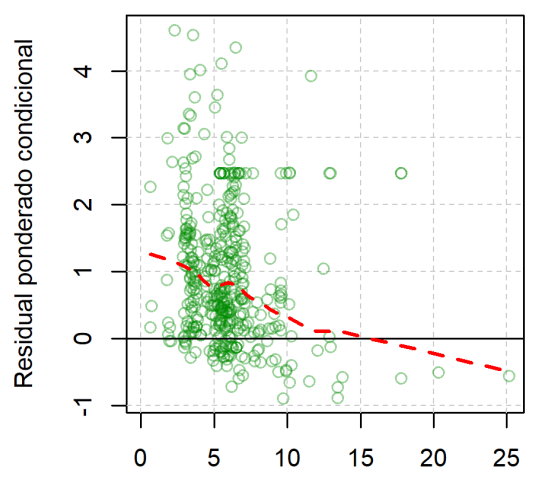

Predicción poblacionales, $\mathrm{mg} / \mathrm{L}$

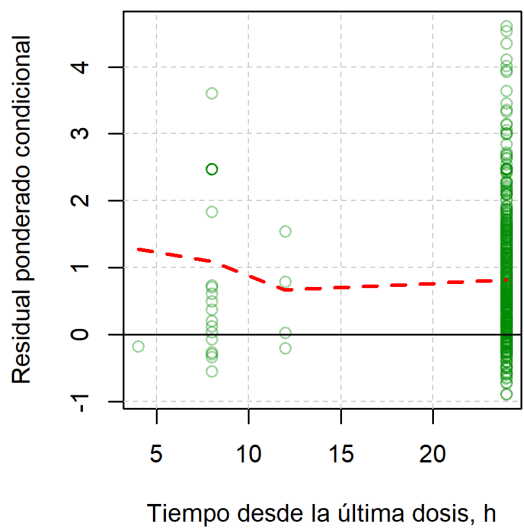

Ediciones Universidad de Salamanca / @(ब@

FarmaJournal, vol. 5, núm. 2 (2020), pp. 15-26 
FiguRA 3. Concentraciones observadas (OBS) y predichas individuales (IPRED) de $\mathrm{DPH}$ estratificadas por las variables incluidas en el modelo final

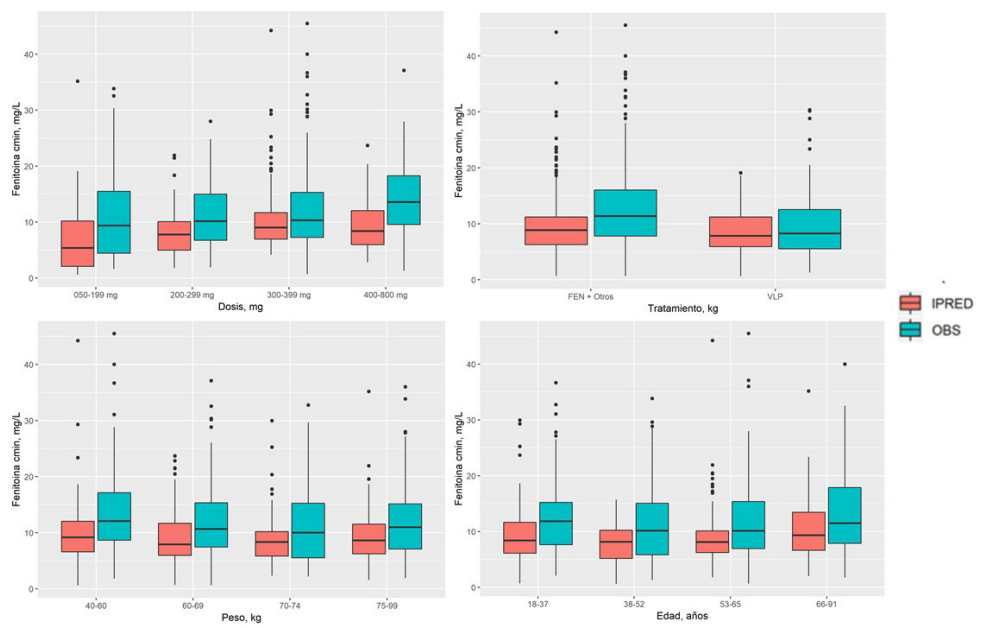

FIGURA 4. Influencia de las variables incluidas en el modelo final de DPH
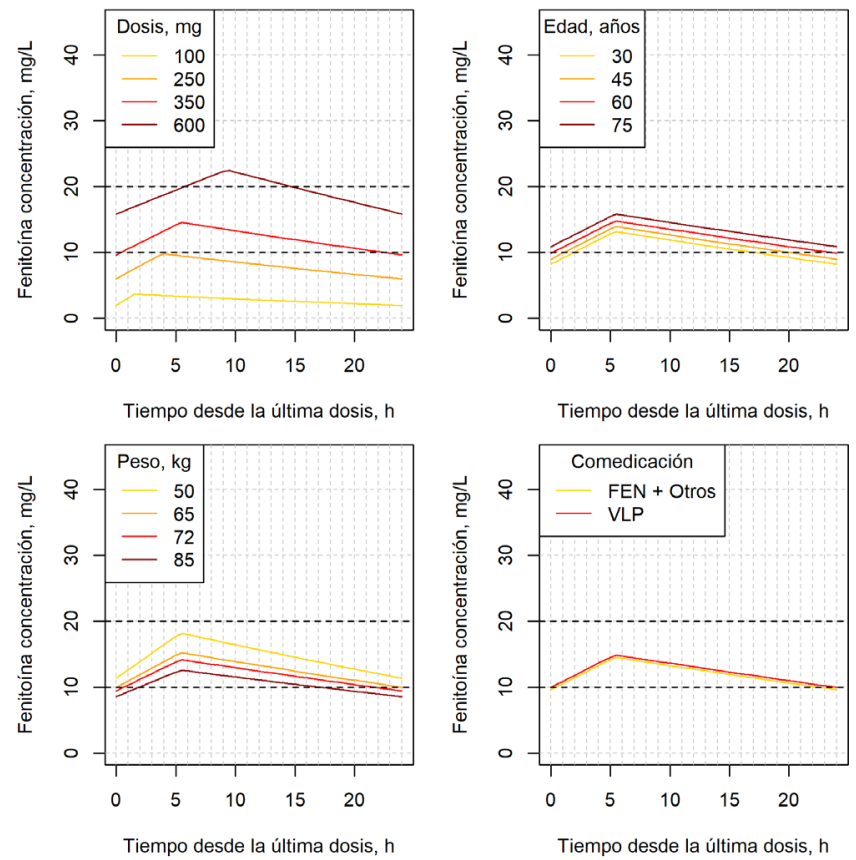

Ediciones Universidad de Salamanca / @@ FarmaJournal, vol. 5, núm. 2 (2020), pp. 15-26 


\section{Discusión}

Los datos utilizados en este estudio proceden de la monitorización de $\mathrm{DPH}$, por lo que la mayor parte de ellos se encuentran dentro del margen terapéutico habitualmente aceptado para este fármaco (10-20 mg/L). Por ello, se observa una gran dispersión en las dosis administradas para conseguir concentraciones dentro de este margen como consecuencia de la elevada variabilidad interindividual de la farmacocinética de DPH. Ello da lugar al conocido como «efecto TDM» ya que, al aplicar esta estrategia de optimización posológica, los pacientes que eliminan más lentamente recibirán dosis menores que aquellos que lo hacen más rápidamente (Martin et al., 1991). Este efecto puede observarse claramente en la Figura 1: un aumento en la dosis no se traduce en un aumento en las concentraciones ya que la dosis está adaptada a la mayor o menor capacidad de eliminación de DPH por parte de cada paciente. Esta es la razón por la que el modelo PopPK desarrollado se ha estratificado en 4 rangos de dosis, a diferencia de otros análisis farmacocinéticos de $\mathrm{DPH}$ publicados que estiman una única $\mathrm{V}_{\max }$ para este fármaco (Alqahtani et al., 2019; Odani et al., 1996).

El modelo PopPK desarrollado indica que la edad y el peso corporal son covariables que muestran una tendencia a modificar la velocidad de eliminación de $\mathrm{DPH}$. Un incremento de la edad provoca una reducción en la $\mathrm{V}_{\text {max }}$, lo que podría atribuirse a un descenso de la función hepática con la edad, considerando que DPH se elimina fundamentalmente por metabolismo hepático. Por otra parte, el peso se correlaciona positivamente con este parámetro PK, resultado coherente, considerando que DPH es un fármaco liposoluble y ampliamente distribuido en toda la masa corporal, lo que se traduce en un descenso en los niveles plasmáticos de DPH que se correlacionan con un incremento de $\mathrm{V}_{\max }$. Estos resultados son concordantes con los observados en estudios previos (Alqahtani et al., 2019; Yukawa et al., 1990; Grasela et al., 1983), aunque en el presente trabajo no se ha confirmado la significación estadística por lo que serían recomendables estudios adicionales incrementando el tamaño de muestra de la población de análisis.

La comedicación con VLP parece incrementar muy ligeramente las concentraciones de DPH, lo que concuerda con el conocido carácter inhibidor enzimático que presenta este fármaco (Bauer, Bloin, 1982; Dudson 1982). No obstante, a pesar de su inclusión en el PopPK su impacto en la cinética de esta población es mínimo y sin relevancia clínica, como se refleja en la Figura 4.

El desarrollo de modelos PopPK resulta muy útil como herramienta para la optimización posológica de fármacos que, como la DPH, presentan una elevada variabilidad interindividual en su farmacocinética; esto es así porque permite su implementación en programas de farmacocinética clínica que, junto con algoritmos bayesianos, permite reducir el número de muestras necesarias para caracterizar el comportamiento cinético de este fármaco en cada paciente. Existen pocos 
estudios que caractericen el comportamiento farmacocinético poblacional de DPH debido a la complejidad de su cinética y aún menos en población caucásica como la nuestra, por lo que el modelo PopPK propuesto en este trabajo puede ser de interés para su aplicación en los programas de TDM implantados en numerosos centros hospitalarios de nuestro país.

\section{Conclusiones}

Se ha desarrollado un modelo farmacocinético poblacional para la fenitoína administrada por vía oral en pacientes adultos con diferentes diagnósticos, utilizando datos procedentes de la monitorización de sus concentraciones en estado de equilibrio. La edad, el peso y la magnitud de dosis han mostrado influencia estadística y clínicamente significativa sobre su velocidad de eliminación, reduciendo su variabilidad interindividual de forma significativa. No obstante, se precisa su aplicación en el contexto clínico para confirmar su validez.

\section{Bibliografía}

Alqahtani S, Alzaidi T, Alotaibi M, Alsultan A. Estimation of phenytoin pharmacokinetic parameters in saudi epileptic patients. Pharmacology. 2019; 104(1-2):60-66.

Bauer LA, Blouin RA. Age and phenytoin kinetics in adult epileptics. Clin Pharmacol Ther. 1982; 31(3):301-304.

Beal SL, Sheiner LB, Boeckmann AJ et al. NONMEM 7.1.0 Users 560 Guides. Ellicott City, MD: Icon Development Solutions; 2009

Dodson WE. Nonlinear kinetics of phenytoin in children. Neurology. 1982; 32(1):42-48.

Eadie MJ. Therapeutic drug monitoring-antiepileptic drugs. Br J Clin Pharmacol. 2002; 46(3):185-193.

Grasela TH, Sheiner LB, Rambeck B, Boenigk HE, Dunlop A, Mullen PW et al. Steadystate pharmacokinetics of phenytoin from routinely collected patient data. Clin Pharmacokinet. 1983; 8(4):355-364.

Gugler R, Manion CV, Azarnoff DL. Phenytoin: pharmacokinetics and bioavailability. Clin Pharmacol Ther. 1976; 19(2):135-142.

Hvidberg EF, Dam M. Clinical pharmacokinetics of anticonvulsants. Clin Pharmacokinet. 1976; 1(3):161-188.

Martin ES, Crismon ML, Godley PJ. Postinduction Carbamazepine Clearance in an Adult Psychiatric Population. Pharmacotherapy. 1991, 11(4):296-302.

Odani A, Hashimoto Y, Takayanagi K, Otsuki Y, Koue T, Takano M et al. Population pharmacokinetics of phenytoin in Japanese patients with epilepsy: analysis with a dose-dependent clearance model. Biol Pharm Bull. 1996;19(3):444-448.

R Core Team. R: A language and environment for statistical computing. Viena, Austria: R Foundation for Statistical Computing; 2018. 
Richens A. Clinical pharmacokinetics of phenytoin. Clin Pharmacokinet. 1979; 4(3):153-169. Vajda FJ, Eadie MJ. The clinical pharmacology of traditional antiepileptic drugs. Epileptic Disord. 2014; 16(4):395-408.

Winter ME. Phenytoin. En: Koda-Kimble MA (editor). Basic clinical pharmacokinetics.

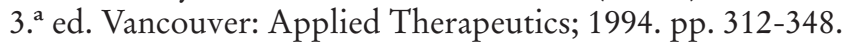

Yukawa E, Higuchi S, Aoyama T. Population pharmacokinetics of phenytoin from routine clinical data in Japan: an update. Chem Pharm Bull (Tokyo). 1990; 38(7):1973-1976. 